\title{
Who are the assistant cooks at the community child centers in South Korea? Focus group interviews with workfare program participants
}

\author{
Jiyoung Park ${ }^{1}$, Chongwon Park ${ }^{2}$, Sanghee Kim ${ }^{3}$, Gill A. Ten Hoor ${ }^{4}$, Gahui Hwang ${ }^{5}$, Youn Sun Hwang ${ }^{6}$ \\ ${ }^{1}$ Assistant Professor, College of Nursing, Institute for Health Science Research, Inje University, Busan · Graduate Student, School of Social Welfare, Yonsei \\ University, Seoul; ${ }^{2}$ Professor, Department of English Language and Literature, Pukyong National University, Busan; ${ }^{3}$ Associate Professor, College of Nursing, \\ Institute for Health Science Research, Inje University, Busan, Korea; ${ }^{4}$ Assistant Professor, Department of Work and Social Psychology, Maastricht University, \\ Maastricht, Netherlands; ${ }^{5}$ Graduate Student, College of Nursing, Yonsei University, Seoul; ${ }^{6}$ Assistant Professor, Department of Nursing, Division of Health \\ Science, Dongseo University, Busan, Korea
}

Purpose: Community child centers (CCCs) were introduced to provide after-school activities and care, including meal services to children from low-income families. The assistant cooks, who have the main responsibility for making and serving food at CCCs, are a major factor influencing the eating habits of children using CCCs. In this study, we tried to identify and understand who the assistant cooks are, what their job responsibilities are, and what they need in order to be able to provide children with healthy meals. Methods: Three focus group interviews were held with 17 workfare program participants who worked as assistant cooks at CCCs, and content analysis methods were applied using the NVivo 12 qualitative data analysis software. Results: The assistant cooks reflected on their perceptions of the children's health at the CCCs, their own cooking style, and their role at the CCCs. Additionally, barriers to the optimal provision of their services were pointed out, and improvements were suggested. Conclusion: The results of this study can be used as a fundamental resource for the development of tailored interventions that consider a child's unique environment to address health disparities, specifically with respect to childhood obesity.

Key words: Eating behavior; Focus groups; Health status disparities; Pediatric obesity

\begin{abstract}
Corresponding author Youn Sun Hwang https://orcid.org/0000-0002-7338-6317

Department of Nursing, Division of Health Science, Dongseo University, 47 Jurye-ro, Sasang-gu, Busan 47011, Korea

TEL +82-51-320-4825 FAX +82-51-320-2721

E-MAIL yshwang@gdsu.dongseo.ac.kr

*This study was supported by a National Research Foundation of Korea (NRF) grant funded by the Korean government (MSIP) (No. 2015R1C1A1A01052892).
\end{abstract}

Received Sep 11, 2020 Revised Sep 28, 2020 Accepted Oct 8, 2020
(3) This is an Open Access article distributed under the terms of the Creative Commons Attribution NonCommercial License (http://creativecommons.org/licenses/by-nc/4.0/) which permits unrestricted noncommercial use, distribution, and reproduction in any medium, provided the original work is properly cited.

\section{INTRODUCTION}

Globally, childhood obesity is rapidly increasing among children from families with low socioeconomic status [1]. Unhealthy eating behaviors influence obesity in children from low-income families [2,3]. These unhealthy eating habits include skipping breakfast, picky eating, high-calorie food intake, and consuming less than the recommended amount of fruit and vegetables [4,5]. Contrarily, healthy eating habits are critical for the growth and development of children. They have been shown to improve children's overall health, including mental health and quality of life, and to decrease children's chances of developing obesity and chronic disease [6].

Children from low-income families were found to be more likely to develop chronic diseases than children from high-income families [7], and children with lower economic levels showed undesirable health habits and obesity [8,9]. In addition, atopy and asthma, which are representative chronic diseases of children, were found to be more frequent in children from low-income families [10]. As such, children from low-income families are naturally exposed to health inequalities due to their families' economic situation and environment, and 
community child centers (CCCs) intervene in this situation in South Korea [11].

CCCs in Korea, funded by the Korean Ministry of Health and Welfare, are part of the health policy for vulnerable children and children with disabilities. At CCCs, after-school activities and care, including learning, mentoring, and meal services, are provided based on the community, especially for children from low-income families [11]. These facilities serve a similar function to 21st-century after-school programs in the United States, which provide poor and underperforming students with opportunities to engage in various academic, social, and physical activities after school [12]. CCCs started operation as private programs run by non-governmental organizations (or non-profit organizations). After they were designated as formal child welfare facilities by law in 2004, the number of CCCs has grown to approximately 4,000 centers, and more than 100,000 low-income children use such centers [13].

Meal services at CCCs include box meals, catering services, and cooking directly at the center. The type of meal services differs across centers depending on their circumstances. However, many centers agree on the importance of directly preparing hot meals at centers and therefore hire assistant cooks to prepare these meals [14]. The assistant cooks are low-income workfare program participants in South Korea who are hired to cook at CCCs in order to provide meals to the children while simultaneously being employed in socially stable jobs. However, they have no particular expertise in nutrition and cooking. In addition, a recent study by Kwon and Yeoh [15] showed that the food served at CCCs did not meet the current dietary and nutrition standards for growing children in Korea. Slightly more than half of the centers (55.7\%) had the facilities to systematically provide average-to-good quality food, but a lack of expertise and frequent employee turnover of assistant cooks at CCCs were repeatedly mentioned as problems [16,17].

The influence of CCCs in shaping better eating behaviors in children from low-income families in South Korea should not be overlooked. In general, many children from poor families spend most of their time at a CCC. As these children consume a majority of their meals there (a service that was rated as being of high priority by both the parents and children who chose to use the CCCs) [18], these centers are an important outlet for health and behavior-change interventions. As a first step to improve healthy food practices at CCCs, it is necessary to identify all stakeholders related to the health of children who attend CCCs and to understand all barriers and facilitating factors that may affect the provision of healthy food. We performed a needs assessment with stakeholders, including obese children and their primary caregivers and service providers at centers, to identify these problems $[17,19]$. In partic- ular, a commonly mentioned problem was the assistant cooks' lack of skills or knowledge and responsibility with respect to the importance of their role, which is directly related to the health and quality of the meal service [17]. However, no study has yet investigated the role played by assistant cooks at CCCs, how they cook and prepare meals, and what barriers and facilitating factors they face. Therefore, the current study carried out focus group interviews to identify and understand who the assistant cooks are, their job responsibilities, and what they need in order to be able to provide children with healthy meals.

\section{METHODS}

\section{Participants and Procedure}

Seventeen assistant cooks currently working at CCCs participated, all of whom were women. Their average age was 57 years ( \pm 5 ; range, $46-64$ years), and seven of them (41.2\%) were married. Seven of them (41.2\%) had completed less than middle school, and the remaining 10 (58.8\%) had graduated high school. They had participated in the workfare program for an average of 47 months ( \pm 31 ; range, $8-120$ months), during which they had worked at CCCs for an average of 26 months $( \pm 14$; range, 3-42 months).

Data collection was conducted between November and December 2017. The participants were reminded of the interview via a text message a day before the interview date. At the start of the interviews, the assistant cooks completed a short demographics questionnaire. Each interview took place in a meeting room that was independent from the CCC facilities to allow the assistant cooks to communicate about their experiences at the center more directly and vividly. Light refreshments and snacks were prepared to create an atmosphere conducive to comfortable conversation. Each focus group interview took between 1.5 and 2 hours. The whole discussion was recorded with permission from the participants.

The moderator (principal investigator) of the study has been developing obesity prevention program for low-income children, and the four researchers involved in data analysis visited the CCCs several times before the focus group interviews to increase their understanding of the assistant cooks' circumstances. The moderator encouraged the participants to talk about their experiences, in particular by using the pause and the probe, which are two essential techniques of focus groups [20]. An assistant researcher observed and documented the atmosphere of the focus group and the participants' nonverbal responses and attitudes. The interview questions consisted of semi-structured and open-ended questions, as follows: 
- Why do you think that many of the children visiting CCCs are obese?

- What do you think is a healthy meal for children?

-What are the barriers or facilitating factors that affect you the most when you work at the center?

- In what areas do you want to receive more support to better fulfil your role?

Upon completion of the focus group, the participants received a small participation fee as a token of gratitude for participating in the research.

\section{Ethical Considerations}

This study was carried out after receiving approval from the Institutional Review Board (IRB) at the principal investigator's university (2016-08-003-003). With the help of a public office in the community, 17 assistant cooks who were currently working at CCCs and wished to participate in the interview were recruited. Prior to each interview, the participants were informed about the study topic, purpose, and procedures. Prior to obtaining written informed consent for voluntary participation, it was explained that the interview content would be kept confidential, that anonymity of the participants was guaranteed, and that the data in the study would not be used for any purpose other than research. Participants were allowed to withdraw from the study at any time without giving a reason.

\section{Analytic Approach}

This study adopted a single-category design [20] where one type of participant (i.e., assistant cooks) was the primary focus of the investigation. At least three or four groups are suggested to reach theoretical saturation [20]. Three researchers including the same moderator and assistant researcher conducted three focus group discussions with 17 assistant cooks until adequate saturation was reached. The processes of data entry, analysis, and interpretation, as well as the final writeup, were carried out using NVivo 12 (NVivo 12, QSR International, Massachusetts, US). The data were coded and subdivided into 143 themes. Subsequently, these themes were categorized into six overarching units. To explore participants' perspectives, an open coding method that offered researchers the space to develop codes via collaboration with the participants was used, resulting in naturally emerging themes from the raw data. This led to the establishment of a theory that explicates how the selected assistant cooks perceive themselves [21]. As a reporting tool, the Consolidated Criteria for Reporting Qualitative Research (COREQ) was used [22]. In addition, participant checking was done with one assistant cook who participated in the interview to receive feedback on the final results. In this process, she absolutely agreed with the findings.

\section{RESULTS}

Seventeen participants voiced their perspectives on (1) causes of childhood obesity, (2) healthy eating, (3) assistant cooks' roles, (4) assistant cooks' cooking styles, (5) possible barriers, and (6) facilitating factors that might enable the assistant cooks to provide healthy meals. A detailed summary of the findings can be found in Table 1. In this section, the subdomains that were repeatedly mentioned in the focus groups are presented in detail.

\section{Perceptions of the Causes of Childhood Obesity}

\section{1) Children's eating habits}

Thirteen assistant cooks attributed childhood obesity to children's poor eating habits such as overeating, eating too much salty food, an unbalanced diet, meat-eating, and eating too much instant food. One assistant cook lamented: "Our children in the center are getting heavier every day. I can tell the difference. They eat too many sweets with an unbalanced diet both at home and at school. I try not to give them too much salty food. However, that does not always work."

\section{Perceptions of Healthy Eating}

\section{1) Providing meals in a sanitary manner}

With respect to providing meals in a sanitary manner, 10 participants voiced their perspectives. If the children get sick from food poisoning, the assistant cooks believed that it was because of the food the children ate at the center. To prevent that from happening, the assistant cooks make sure that the food ingredients and the utensils were thoroughly disinfected and emphasized sanitation as their top priority. One cook stated: "The spoons and the chopsticks are disinfected in boiling water three times a week. Also, I always heat up some water to mix with the dishwashing detergent before washing the dishes. I use hot water to wash the dishes even in the summer when it makes me sweat. I wash dishes like that in all of the seasons."

Another cook stated the following about inspections: "When I first received an inspection, I saw how some of the cotton swabs turned red and others turned yellow. That's when I realized that I need to clean everywhere, including the outside. From then on, I cleaned the outside as well."

A third assistant cook presented her experience on regular training by saying: "The trainings were about how to use the 
Table 1. A Summary of the Themes Identified in the Study $(N=17)$

\begin{tabular}{|c|c|c|}
\hline Domains & Subdomains & $\mathrm{n}(\%)$ \\
\hline $\begin{array}{l}\text { Perceptions of the causes of } \\
\text { childhood obesity }\end{array}$ & $\begin{array}{l}\text { Children's eating habits* } \\
\text { Parental negligence in caring for the children* } \\
\text { Center's early meal times to encourage additional meals at home* } \\
\text { Stress from home/home situation } \\
\text { Center children's addiction to mobile phones }\end{array}$ & $\begin{aligned} 13 & (76.5) \\
5 & (29.4) \\
4 & (23.5) \\
1 & (5.9) \\
1 & (5.9)\end{aligned}$ \\
\hline Perceptions of healthy eating & $\begin{array}{l}\text { Providing a meal in a sanitary manner* } \\
\text { - Inspection focused on sanitation* } \\
\text { - Training focused on sanitation* } \\
\text { Letting the children eat the foods they like in large amounts* } \\
\text { Using healthy food ingredients* } \\
\text { Making the children eat fish and vegetables* } \\
\text { Never thinking about healthy eating } \\
\text { Limiting the amount of food children consume } \\
\text { Not using artificial flavor enhancers } \\
\text { Following the given menu } \\
\text { Using less oil when cooking }\end{array}$ & $\begin{array}{r}10(58.8) \\
8(47.1) \\
7(41.2) \\
9(52.9) \\
6(35.3) \\
5(29.4) \\
2(11.8) \\
2(11.8) \\
2(11.8) \\
2(11.8) \\
1(5.9)\end{array}$ \\
\hline Assistant cooks' roles & $\begin{array}{l}\text { Center mom* } \\
\text { - Provide general care like a mom* } \\
\text { - Provide home-style food having its own unique style and taste* } \\
\text { Assistant to the center director and the social workers* }\end{array}$ & $\begin{array}{r}14(82.4) \\
11(64.7) \\
7(41.2) \\
4(23.5)\end{array}$ \\
\hline Assistant cooks' cooking styles & $\begin{array}{l}\text { Cooking my own way* } \\
\text { Searching and using existing recipes on the internet* }\end{array}$ & $\begin{array}{l}5(29.4) \\
4(23.5)\end{array}$ \\
\hline Barriers & $\begin{array}{l}\text { Perceived role conflicts among centers due to the unclear job specification* } \\
\text { Low wages* } \\
\text { Poor working conditions* } \\
\text { Uneven budget expenses* } \\
\text { Religious pressure from the center director* } \\
\text { Unrealistic expectancies from the center director* } \\
\text { Discontent with respect to laborious cooking* } \\
\text { No authority to cook food independently as assistant cooks } \\
\text { Health problems (e.g., menopause, heart disease) } \\
\text { Unexpected sanitary audit }\end{array}$ & $\begin{array}{l}9(52.9) \\
6(35.3) \\
6(35.3) \\
3(17.6) \\
3(17.6) \\
3(17.6) \\
3(17.6) \\
2(11.8) \\
2(11.8) \\
1(5.9)\end{array}$ \\
\hline Facilitators & $\begin{array}{l}\text { Assistance from the members* } \\
\text { Cooking confidence* } \\
\text { Small amount of cooking* } \\
\text { Positive reinforcement regarding the work* } \\
\text { Active communication among assistant cooks regarding the work } \\
\text { An authority to cook food independently as assistant cooks }\end{array}$ & $\begin{array}{l}6(35.3) \\
5(29.4) \\
4(23.5) \\
3(17.6) \\
2(11.8) \\
1(5.9)\end{array}$ \\
\hline
\end{tabular}

*Cited more than three times in the focus groups; therefore, some of these points are discussed in detail in the main manuscript.

refrigerator properly, how to manage frozen food, what needs to be done after washing the dishes, ways of disinfecting, and so on. The trainings were good."

\section{2) Letting the children eat the foods they like in large amounts}

Nine assistant cooks thought that it was healthy for the children to eat large amounts of food because the children usually eat a lot when they are served foods they enjoy, such as meat and fried food, but do not eat well when foods they dislike, such as vegetables, are served.

One assistant cook stated: "The kids don't eat properly when they are at home. Therefore, it is better to have a lot of their favorite items here. If I see something on the menu that the kids don't like, I tell the director that the kids don't like it. Then, I ask her if I can change it to something the kids like and go ahead and change it."

\section{3) Using healthy food ingredients}

Six assistant cooks pointed out the importance of using healthy food ingredients. One assistant cook stated: "I believe that frozen food is harmful for the children's health, and I don't want to give it to the children when possible." 


\section{Assistant Cooks' Roles}

\section{1) Center moms}

Fourteen participants extended their roles and identified themselves as "center moms". CCCs have a "center mom", just like there is a "mom" in each household. As "center moms", the assistant cooks took charge of the children's overall health, providing them with warm meals they personally made, playing with them when they had the time, and washing them to ensure they were clean.

(1) Providing general care like a mom

Regarding the possible roles of assistant cooks, 11 participants emphasized the importance of providing overall care for the children, including being aware of what food each child likes and dislikes, protecting the children's health by being aware of foods that a child is allergic to (to avoid this when cooking), playing with the children when the teachers are absent, and washing them or shampooing their hair when needed.

One assistant cook stated, "They have their own mom at home, but the moms don't wash their kids' hair and just send them here. Their hair stinks and can become all scruffy. Then, I must wash them. I keep noticing the things that they need because I don't think of them as someone else's kids."

(2) Providing home-style food with its own unique style and taste

Rather than using commercial ready-made meals, seven service providers thought that it was important to personally prepare warm meals and to serve them on time with the mind-set that they were providing meals for their own family members.

One cook stated: "The children really don't eat the boxed meals that are purchased. I guess they can tell the difference between boxed meals and the meals I personally make for them."

Another cook also added by saying, "The centers need to have a cook like me to prepare and serve a meal right on time."

\section{Assistant Cooks' Cooking Styles}

\section{1) Cooking my own way}

Five assistant cooks cooked the food their own way, as they believed that the children would enjoy the food if it is made to suit their own taste without using any special methods.

One assistant cook said: "I just do it my way. We don't keep any MSG (monosodium L-glutamate) in the kitchen. So, I think about ways to make the food taste good without using MSG. I try my best to make the food suit my own tastes because I think if it tastes good to me, it will taste good to the children as well."

\section{2) Searching and using existing recipes on the internet}

In addition, some participants mentioned that they used the internet or applications on their phone to find existing recipes when they had to cook a dish for the first time, because an accredited institution (e.g., a public health center) provides the menu, but does not provide a way to make it.

One cook said: "There are a lot of dishes that are new to me. I look on the internet for them." Another cook also added: "If there is a dish I don't know, I look up the recipe on Naver [the name of a representative portal site in South Korea]."

\section{Barriers}

\section{1) Perceived role conflicts among centers due to the unclear job specification}

With respect to barriers, nine assistant cooks were confused about their roles assigned at the different centers. According to them, cleaning, recycling, and making kimchi (a traditional side dish made from salted and fermented vegetables), were not part of the assistant cooks' role, although some assistant cooks made kimchi, whereas others do not make it at all. They highlighted a lack of consistency in doing the job.

Their perceived fundamental role was to cook and to have conversations with the children like a center mom. The assistant cooks' job specifications were unclear based on the inconsistent rules that they were forced to follow across the centers and frequent substitutions of center directors and teachers.

One assistant cook lamented: "Our center director and a teacher have been changed, and no one knows the rules to manage the center. As a result, it might be hard for the new center director to manage the staff to do the right job. Because the center director changed, I have to arrive by 12:30 to clean the center."

\section{2) Low wages}

The relatively low wages influenced the performance of the assistant cooks. Six participants in the study believed that their salary was relatively low considering the amount of work they had to do every day.

One assistant cook stated, "A public official informed me that our wages are low. He said that the Department of Health and Humans Services decided the salary for workfare program participants. If the salary for assistant cooks increases, healthy (more skilled) people will apply for this job."

\section{3) Poor working conditions}

Six participants in the study raised the issue of inadequate working conditions related to the facilities, including heating 
power, frying pans, refrigerators, heaters, air conditioning, and the hood.

One cook described her experience of cooking naengmyon (a cold noodle dish) by stating, "Every time I cook naengmyon, I cannot cook it in a single session because our heating power setup is for domestic purposes, which means that we cannot cook much food at the same time. Considering the number of children, cooking naengmyon at our center takes a million years."

Another cook added, "Our ppokunbap (fried rice) is always soft and wet. The reason is that the pan that we are using is made of nickel silver. If p pokunbap were cooked using a stainless steel-coated pan that would never happen. Recently, we bought a new frying pan, which is the only cooking appliance that we changed throughout the whole year."

A third assistant cook raised the issue of the air circulation in their working places. She lamented: "(..) Where I work, there are no windows and no hood, and, in the narrow space, I absorb everything there."

\section{Facilitators}

\section{1) Assistance from the members}

Six participants noted that help from the center director and social workers was a facilitating factor in helping the assistant cooks.

One assistant cook said, "( $\cdots)$ In our case, the center director bought everything for us. He bought everything from the market, and, if we needed, I wrote down the list and contacted the director in his office, and he usually went to the market. I didn't have to do anything else, and I am very satisfied with it."

\section{2) Cooking confidence}

Five participants showed confidence in their cooking abilities. One assistant cook stated, "I like cooking at home. I am very comfortable cooking. Everything is in my brain, and, based upon my inclinations, I prepare food and serve. There is no need to ask other people. I cook as I like."

\section{DISCUSSION}

In this study, we aimed to understand assistant cooks, who influence the eating habits of children from low-income families who attend CCCs. In addition, we examined the characteristics of workfare program participants working in the child welfare system and discussed the implications of their effects on children's and youth's eating behaviors. Understanding the assistant cooks' needs, attitudes, and barriers might help in developing interventions to improve children's eating habits at the CCCs and beyond.

\section{Where Do the Attitudes of the Assistant Cooks Come from?}

First, with respect to children's obesity and dietary habits, the assistant cooks' thoughts on the causes of childhood obesity and healthy eating were examined. Most assistant cooks in the study stated that poor dietary habits, including overeating and an unbalanced diet, are among the main causes of child obesity at CCCs, which is already widely known [23]. In other words, they recognized the importance of healthy eating habits for childhood obesity. Regarding their perceptions of healthy eating, several assistant cooks agreed that healthy eating means eating healthy foods made with fresh ingredients (as opposed to frozen foods) and eating fish and vegetables. However, they also described some elements of what they believed healthy eating to be from the food provider's point of view, such as maintaining sanitation and providing many foods that children enjoy, such as meat and fried food. Differences in perceptions of healthy eating are influenced by socioeconomic factors, education, and other internal and external factors. Namely, household economic status is a key factor that influences eating behaviors (i.e., how people eat, cook, and serve foods) [24]. The assistant cooks in the study were workfare program participants, who represent a low-income class in South Korea. The economic status of low-income individuals may hinder their ability to understand healthy eating [25].

Another reason that many assistant cooks defined healthy eating as including the provision of food in a sanitary fashion may be that sanitation was heavily emphasized in their line of work. CCCs are government-funded organizations that are supervised and are subject to sanitary inspections by local district offices. During these health inspections, CCCs are checked for the disinfection and management of tableware and cooking utensils, food storage practices, and the salinity of cooked food. Thus, frequent health inspections and relevant education were important to the assistant cooks, and the intense focus on sanitary practices may have caused them to develop skewed perceptions of healthy eating. However, in this study, we attempted to explore the assistant cooks' perceptions of healthy eating, and additional research on factors affecting their attitudes and misperceptions is needed.

An interesting finding in our study was that there was a stark contrast between assistant cooks' and others' perspectives on their performance. Other CCC workers, parents, and the national agency that manage assistant cooks described them as incompetent participants of a workforce program who lacked responsibility [17]. This type of classification may stigmatize those who participate in workforce programs. However, in this study, the assistant cooks actually perceived 
themselves to be handling responsibilities beyond the expected scope of their duties, thinking of themselves as "center moms" when they helped children with things other than preparing food. This difference may be attributable to various factors, one of which is a lack of understanding of the assistant cooks' roles and responsibilities. For instance, parents and CCC workers identified assistant cooks as significant contributors to CCC children's obesity rates [17], while in this study, assistant cooks tended to blame parents and CCC workers for the children's obesity. This type of finger-pointing may undermine mutual trust, and such conflict between stakeholders may adversely impact children from low-income families. Thus, assistant cooks must be able to share more about their roles and channels through which stakeholders can regularly communicate with one another. In addition, collaboration among various stakeholders including parents, service providers at CCCs, and community nurses directly working with low-income children is essential to prevent and manage childhood obesity in low-income children.

\section{How Can We Improve Healthy Eating at the Commu- nity Child Centers?}

Most assistant cooks said that they cook with their own recipes, which simply meant that they do not use MSG and cook to suit their own palate. Furthermore, the menus at CCCs are planned according to children's tastes, so sometimes the assistant cooks must prepare unfamiliar foods. In such cases, assistant cooks said that they use recipes taken from the internet. However, these recipes are usually written using simple, easy-to-understand language, so it is difficult to determine whether the food meets the healthy eating standards for children. Such non-standardized cooking in group food services may adversely impact children's healthy dietary habits, as the assistant cooks' perceptions of healthy eating often do not coincide with contemporary nutritional knowledge, probably because they have not formally learned about cooking and nutrition. Education programs are required to teach assistant cooks how to prepare and serve standardized food at CCCs to modify their habits and improve their skills and awareness of healthy eating. It is also crucial to develop and promote official cooking websites to help them resolve any issues or difficulties that they experience when cooking. In addition, standardized meal preparation guidelines should be developed to help assistant cooks, who are non-experts, to help children at CCCs to form healthy eating habits.

Without a clear job description, assistant cooks are confused about the content and scope of their work, which can cause conflict with their service providers. One notable finding in this study was that assistant cooks believed that clean- ing, recycling, and making kimchi was not within the scope of their work, and this was therefore a point of conflict. Although kimchi is food, in South Korea, making kimchi is not treated the same as preparing other food as it is generally prepared through several steps over a long time and requires relatively intense labor. Thus, assistant cooks may consider making kimchi to be unreasonably burdensome as they are temporary workers participating in a workforce program. These workers are likely paid a relatively low wage for this reason and are working conditionally to receive the national basic livelihood security, so they tend to look for easier work. Furthermore, the assistant cooks examined in this study had a relatively low average education level, with about $60 \%$ being high school graduates and $40 \%$ being middle school graduates or lower. Given these factors, assistant cooks may be more conservative with regard to the effort that they want to put into their work. Thus, a clear, written job description should be provided to assistant cooks when they are hired to provide them with a full understanding of their duties. Moreover, assistant cooks mentioned several inconveniences in their cooking environment, which reduced their morale. In order to motivate the assistant cooks and improve their job performance, they must be provided with a pleasant work environment, adequate space, proper tools, clear responsibilities, and workloads that are appropriate for workers in their situations.

Assistant cooks stated that the self-esteem that they attained from cooking was a motivating factor in their work. Self-esteem is a psychological term that refers to a person's assessment of and attitude toward their own abilities and value, and it contributes to the alteration and maintenance of behaviors [26]. Thus, it is important to develop and implement strategies for increasing assistant cooks' self-esteem through education programs, which they requested. In fact, in this study, they also asked for a cooking class to learn how to cook food. This would help improve their knowledge and skills and increase their confidence in cooking. The assistant cooks also said that they needed to attend regular gatherings of assistant cooks because they had few opportunities to connect with others and would like to do so to receive social support. Social support includes various forms of aid obtained from significant others, such as family members, friends, colleagues, or experts, and it plays a critical role in maintaining and promoting personal health [27]. Furthermore, social support increases resilience against stress [28]; therefore, it is important to develop programs to help assistant cooks gain adequate social support from their colleagues.

Since this was the first qualitative study attempting to identify who assistant cooks are and what role they play in general, it was not possible to investigate their perceptions focusing on children's health, health-promoting practices (eating 
behaviors), and relationships with those factors. In addition, as this study explored the perceptions of assistant cooks at CCCs, the perspectives on this issue from other stakeholders surrounding low-income children, including community nurses and schoolteachers, may differ.

\section{CONCLUSION}

It is difficult to provide professional education and interventions for only assistant cooks to improve children's dietary habits. Although enhancing assistant cooks' competence through education is important, the assistant cooks hired by CCCs are not experts with insights into nutritional balance and various cooking methods. The demands placed upon them must also take into consideration their working conditions and the qualifications of the applicant pool from which the assistant cooks are drawn. In this study, most assistant cooks had positive attitudes about cooking at the center and wanted to improve children's health. However, they lacked the knowledge and skills to cook a healthy meal. The central government must develop standardized menus and recipes that assistant cooks can easily follow and offer official nutrition education or cooking classes considering their ability to improve their knowledge, skill, and self-efficacy on cooking. Furthermore, CCCs should secure adequate funds and channels for improving the centers' environments to offer healthy meals to children and to meet assistant cooks' demands. These efforts will ultimately help low-income children develop proper eating behaviors and reduce the risk of chronic diseases by preventing imbalances in nutritional supply and obesity. However, healthy eating habits and adequate nutrition in children can be further strengthened through parental partnerships, as well as changes in assistant cooks and CCCs. In addition, community nurses should make efforts to close the gap in children's health status by continuing to pay attention to these areas in terms of primary prevention for the disadvantaged population.

\section{Conflict of interest}

No existing or potential conflict of interest relevant to this article was reported.

\section{Data availability}

Please contact the corresponding author for data availability.

\section{REFERENCES}

1. Hales CM, Carroll MD, Fryar CD, Ogden CL. Prevalence of obesity among adults and youth: United States, 2015-2016 [Internet]. Jacksonville, FL: Center for Disease Control and Prevention; 2017 [cited 2020 September 11]. Available from:

https://www.cdc.gov/nchs/data/databriefs/db288.pdf

2. Goisis A, Sacker A, Kelly Y. Why are poorer children at higher risk of obesity and overweight? A UK cohort study. European Journal of Public Health. 2016;26(1):7-13.

https://doi.org/10.1093/eurpub/ckv219

3. Pieper JR, Whaley SE. Healthy eating behaviors and the cognitive environment are positively associated in low-income households with young children. Appetite. 2011;57(1):59-64.

https://doi.org/10.1016/j.appet.2011.03.016

4. Park J. Predictors of health-promoting behaviors in children from low-income families: An ecological approach. Nursing and Health Sciences. 2018;20(1):60-68. https://doi.org/10.1111/nhs.12388

5. Ranjit N, Wilkinson AV, Lytle LM, Evans AE, Saxton D, Hoelscher DM. Socioeconomic inequalities in children's diet: The role of the home food environment. International Journal of Behavioral Nutrition and Physical Activity. 2015;12(suppl 1):S4.

https://doi.org/10.1186/1479-5868-12-s1-s4

6. Khan MKA, Faught EL, Chu YL, Ekwaru JP, Storey KE, Veugelers PJ. Is it nutrients, food items, diet quality or eating behaviours that are responsible for the association of children's diet with sleep? Journal of Sleep Research. 2017;26(4):468-476.

https://doi.org/10.1111/jsr.12466

7. Currie J, Mark S. Socioeconomic status and child health: Why is the relationship stronger for older children? American Economic Review. 2003;93(5):1813-1823. https://doi.org/10.1257/000282803322655563

8. Kang HT, Park KH, Kwon YJ, Im HJ, Paek DM, Lee HJ, et al. Study on the relationship between childhood obesity and various determinants, including socioeconomic factors, in an urban area. Journal of Preventive Medicine and Public Health. 2006;39(5):371-378.

9. Drewnowski A, Rehm C, Kao C, Goldstein H. Poverty and childhood overweight in California Assembly Districts. Health and Place. 2009;15(2):631-635. https://doi.org/10.1016/j.healthplace.2008.09.008

10. Sung KS. Development and effect of the group theraplay program for resilience promotion of the children with chronic illness [dissertation]. Seoul: Korea University; 2005. p. 1-112.

11. Park J, Baek S, Im M. Trends in health-related interventions on children attending community child care centers in South Korea. Child Health Nursing Research. 2018;24(2):241-251. https://doi.org/10.4094/chnr.2018.24.2.241

12. Ward C, Gibbs BG, Buttars R, Gaither PG, Burraston B. Assessing the 21st century after-school program and the educational gains of LEP participants: A contextual approach. Journal of Education for Students Placed at Risk. 2015;20(4):312-335.

https://doi.org/10.1080/10824669.2015.1050278

13. National Center for the Rights of the Child. Introduction of 
community child center [Internet]. Seoul: National Center for the Rights of the Child; 2018 [cited 2020 June 30]. Available from: www.icareinfo.go.kr/intro/center/centerAboutus.do?menuNo= 2004300

14. Ministry of Health and Welfare. 2018 Community Child Center Support Program [Internet]. Sejong: Ministry of Health and Welfare; 2017 [cited 2020 February 18]. Available from: www.mohw.go.kr/react/jb/sjb0601vw.jsp?PAR_MENU_ID=03 \&MENU_ID $=03160501 \& C O N T$ SEQ $=343358 \&$ page $=1$

15. Kwon S, Yeoh Y. Status of meal serving and nutritional quality of foods served for children at community child centers in Korea. Journal of the East Asian Society of Dietary Life. 2015;25(2):352-362. https://doi.org/10.17495/easd1.2015.4.25.2.352

16. Kwon S. Needs for improving quality of foodservice management at community child centers in Korea. Korean Journal of Food and Nutrition. 2017;30(4):749-758. https://doi.org/10.9799/ksfan.2017.30.4.749

17. Park J, Ten Hoor GA, Cho J, Kim S. Service providers' perspectives on barriers of healthy eating to prevent obesity among low-income children attending community childcare centers in South Korea: A qualitative study. Ecology of Food and Nutrition. 2020;59(3):311-328. https://doi.org/10.1080/03670244.2020.1722948

18. Suh HJ, Rho SH. Children-youths and parents' selection criteria of community children's center. Korean Journal of Youth Studies. 2013;20(2):167-189.

19. Park J, Ten Hoor GA, Baek S, Baek SH, Lee JY, Lee H. Social ecological barriers for healthy eating of obese children and their caregivers in low-income families in South Korea. Ecology of Food and Nutrition. 2020. Forthcoming.

20. Krueger RA, Casey MA. Focus groups: A practical guide for ap- plied research. 5th ed. Thousand Oaks, CA: Sage Publications, Inc.; 2014. p. 120-121.

21. Saldana J. The coding manual for qualitative researchers. Thousand Oaks, CA: Sage publication; 2009. p. 12.

22. Tong A, Sainsbury P, Craig J. Consolidated criteria for reporting qualitative research (COREQ): A 32-item checklist for interviews and focus groups. International Journal for Quality in Health Care. 2007;19(6):349-357. https://doi.org/10.1093/intqhc/mzm042

23. Centers for Disease Control and Prevention. Childhood obesity causes and consequences [Internet]. Atlanta, GA: Center for Disease Control and Prevention; 2020 [cited 2020 September 11]. Available from: www.cdc.gov/obesity/childhood/causes.html

24. Cwerner S, Gadsby D. Healthy eating: Health behaviours joint strategic needs assessment literature review [Internet]. Preston: Lancashire County Council; 2014. Available from:

https://www.lancashire.gov.uk/media/899796/healthy-eatingupdated-links-oct-2016.pdf

25. Eikenberry N, Smith C. Healthful eating: Perceptions, motivations, barriers, and promoters in low-income Minnesota communities. Journal of the American Dietetic Association. 2004;104(7):1158-1161. https://doi.org/10.1016/j.jada.2004.04.023

26. Rosenberg M. Society and the adolescent self-image. Princeton: Princeton University Press; 1965.

27. Glanz K, Rimer BK, Viswanath K. Health behavior and health education: Theory, research, and practice. 4th ed. San Francisco, CA: Jossey-Bass; 2008. p. 189-207.

28. Watkins K, Hill EM. The role of stress in the social support-mental health relationship. Journal of College Counseling. 2018;21(2): 153-164. https://doi.org/10.1002/jocc.12094 